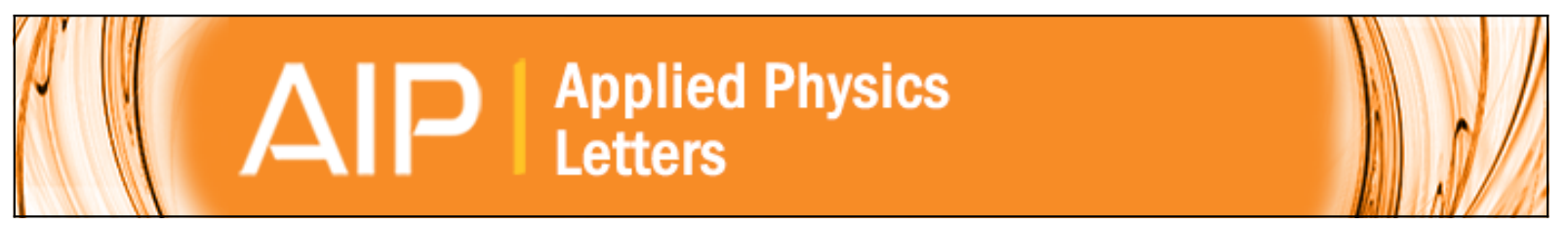

\title{
Doped graphene nanohole arrays for flexible transparent conductors
}

Jianwei Liu, Guowei Xu, Caitlin Rochford, Rongtao Lu, Judy Wu, Christina M. Edwards, Cindy L. Berrie, Zhijun

Chen, and Victor A. Maroni

Citation: Applied Physics Letters 99, 023111 (2011); doi: 10.1063/1.3610939

View online: http://dx.doi.org/10.1063/1.3610939

View Table of Contents: http://scitation.aip.org/content/aip/journal/apl/99/2?ver=pdfcov

Published by the AIP Publishing

\section{Articles you may be interested in}

Optical response of oriented and highly anisotropic subwavelength metallic nanostructure arrays

Appl. Phys. Lett. 102, 201115 (2013); 10.1063/1.4807031

SeZnSb alloy and its nano tubes, graphene composites properties

AlP Advances 3, 042124 (2013); 10.1063/1.4802912

Investigation of thermal conductivity, viscosity, and electrical conductivity of graphene based nanofluids

J. Appl. Phys. 113, 084307 (2013); 10.1063/1.4793581

Photonic assisted light trapping integrated in ultrathin crystalline silicon solar cells by nanoimprint lithography

Appl. Phys. Lett. 101, 103901 (2012); 10.1063/1.4749810

Large-area suspended graphene on $\mathrm{GaN}$ nanopillars

J. Vac. Sci. Technol. B 29, 060601 (2011); 10.1116/1.3654042

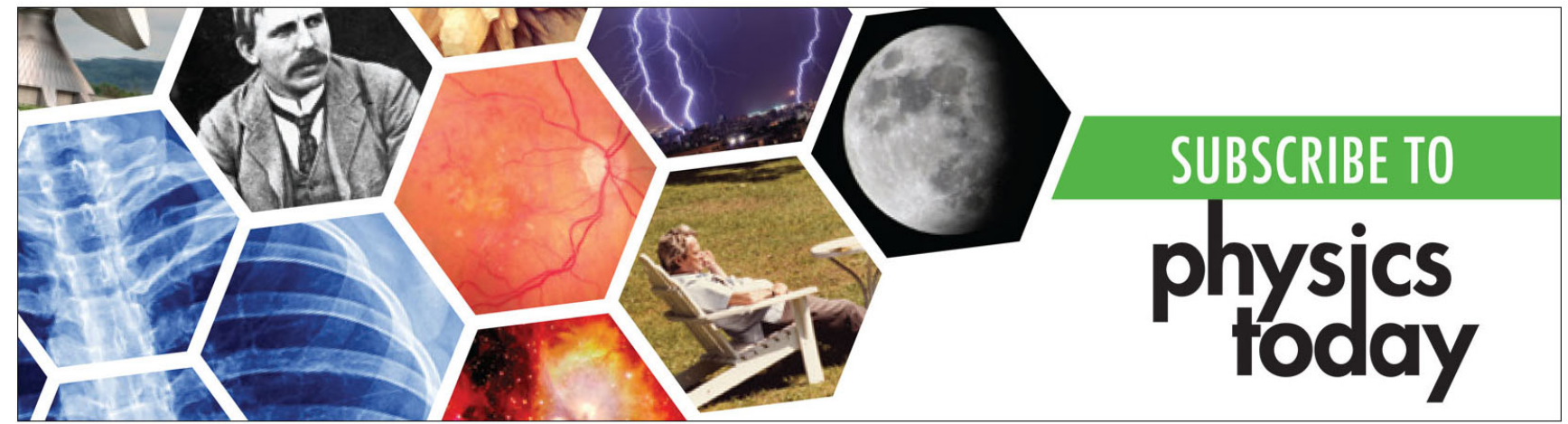




\title{
Doped graphene nanohole arrays for flexible transparent conductors
}

\author{
Jianwei Liu, ${ }^{1, a)}$ Guowei Xu, ${ }^{1}$ Caitlin Rochford, ${ }^{1}$ Rongtao Lu, ${ }^{1}$ Judy Wu, ${ }^{1}$ \\ Christina M. Edwards, ${ }^{2}$ Cindy L. Berrie, ${ }^{2}$ Zhijun Chen, ${ }^{3}$ and Victor A. Maroni ${ }^{3}$ \\ ${ }^{1}$ Department of Physics and Astronomy, University of Kansas, Lawrence, Kansas 66045, USA \\ ${ }^{2}$ Department of Chemistry, University of Kansas, Lawrence, Kansas 66045, USA \\ ${ }^{3}$ Materials Science Division, Argonne National Laboratory, Argonne, Illinois 60439, USA
}

(Received 31 March 2011; accepted 21 June 2011; published online 15 July 2011)

\begin{abstract}
Graphene nanohole arrays (GNAs) were fabricated using nanoimprint lithography. The improved optical transmittance of GNAs is primarily due to the reduced surface coverage of graphene from the nanohole fabrication. Importantly, the exposed edges of the nanoholes provided effective sites for chemical doping using thionyl chloride was shown to enhance the conductance by a factor of 15-18 in contrast to only 2-4 for unpatterned graphene. GNAs can provide a unique scheme for improving both optical transmittance and electrical conductivity of graphene-based transparent conductors. (C) 2011 American Institute of Physics. [doi:10.1063/1.3610939]
\end{abstract}

Graphene ${ }^{1}$ has many unique properties including high charge mobility, high optical transmittance, and flexibility, which make graphene attractive for optoelectronic applications, including solar cells ${ }^{2}$ and transparent conductors. ${ }^{3}$ Currently, indium tin oxide (ITO) is widely employed as a transparent conductor. ${ }^{3}$ However, ITO has several limitations. Indium used in ITO is available in limited amounts. In addition, ITO is brittle and has relatively poor transparency at longer wavelengths. The transmittance of ITO reduces to less than $70 \%$ at wavelengths longer than $750 \mathrm{~nm},{ }^{3}$ which is not favorable for high-efficiency, broad-band photovoltaic device applications.

Graphene may provide a promising alternative to ITO as demonstrated by its high electrical conductivity, ${ }^{4}$ remarkable optical transparency, ${ }^{5}$ and work function of $\sim 4.42 \mathrm{eV}{ }^{6}$ The recent work in large-area growth of graphene using chemical vapor deposition (CVD) on metal foils represents a critical step towards application of graphene for flexible transparent conductors. ${ }^{3}$ A single layer of graphene absorbs only about $2.3 \%$ of incident white light. ${ }^{7}$ The sheet resistance $\left(\mathrm{R}_{\mathrm{s}}\right.$ $=$ resistivity/thickness) of graphene is predicted from the above equation to be around $31 \Omega / \square,{ }^{8}$ which is lower than the experimentally obtained range, from 125 to 10000 $\Omega / \square .{ }^{3,9}$ Even when using the same process such as CVD, ${ }^{10}$ $\mathrm{R}_{\mathrm{s}}$ of graphene varies quite dramatically. ${ }^{11,12}$ One approach for improving $R_{s}$ is to stack multiple layers of graphene. However, adding multiple layers of graphene decreases optical transmission and increases the complexity of transparent conductor fabrication. ${ }^{9}$ Another approach is via chemical doping (CD). CD has been shown to increase the conductivity of carbon nanotubes and graphene by depletion of electrons via hole doping. ${ }^{13,14}$ However, the enhancement is limited to a factor of only 2-3 due to most probably lack of effective sites for molecular attachment.

Graphene nanohole arrays (GNAs) may provide a unique scheme for improving both conductivity and transmittance. In addition to improving the transmittance by reducing graphene coverage, GNAs can provide large number exposed edges of holes for effective $\mathrm{CD},{ }^{15}$ allowing the possibility of tuning

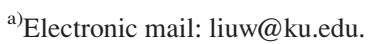

electrical transport properties of graphene. Here, we report on GNAs fabricated using nanoimprint lithography (NIL). The correlation between transmittance and conductivity has been investigated in GNAs in visible spectrum before and after CD. By doping one-layer GNAs using thionyl chloride $\left(\mathrm{SOCl}_{2}\right)$, we were able to obtain $96 \%$ and $98 \%$ transmittance at $550 \mathrm{~nm}$ and $750 \mathrm{~nm},{ }^{24}$ respectively, and $\mathrm{R}_{\mathrm{S}}$ of $286 \Omega / \square$.

The Raman spectrum is shown in Fig. 1 on a graphene sample. The two intense features associated with graphene can be observed in the spectrum: $G$ peak at around 1580 $\mathrm{cm}^{-1}$ and $2 \mathrm{D}$ peak at $\sim 2720 \mathrm{~cm}^{-1}$. The $\mathrm{G}$ peak is due to the doubly degenerate zone center $\mathrm{E}_{2 \mathrm{~g}}$ mode and the 2D peak, the second order of zone-boundary phonons in graphene. ${ }^{16}$ The intensity of $2 \mathrm{D}$ peak is at least 2 times greater than that of $G$ peak and peaks are symmetric, indicating the presence of a single layer of graphene. ${ }^{16,17}$ In addition, the very low intensity D peak was observed on graphene sample, which suggests that defect population in our graphene samples is insignificant. ${ }^{16,17}$ The inset of Fig. 1 depicts a scanning electron microscopy (SEM) image of graphene on a $\mathrm{Cu}$ foil. A continuous layer of graphene with wrinkles represents a typical morphology of CVD graphene on Cu foils. ${ }^{11}$

Figs. 2(a) and 2(b) depict SEM of GNAs with an ordered hexagonal pattern of holes before and after the NIL resist was removed. The diameter of holes is $245 \pm 15 \mathrm{~nm}$ and the

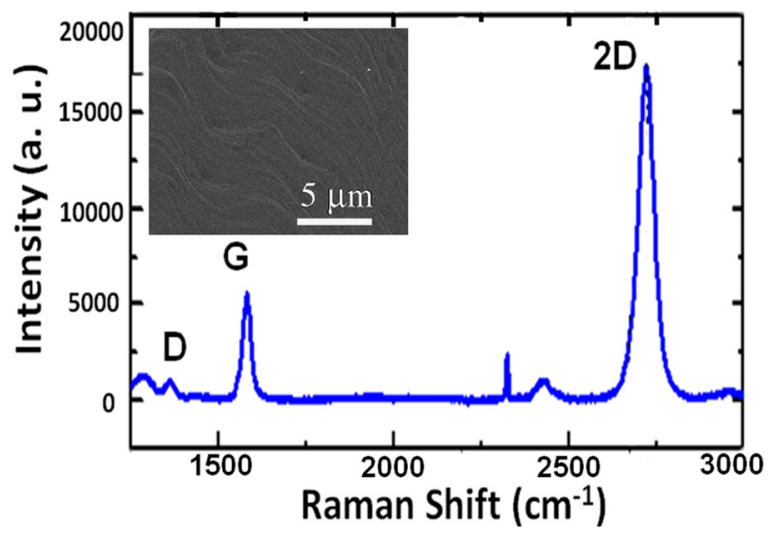

FIG. 1. (Color online) Raman spectrum of graphene on copper foil; inset: SEM image of graphene grown on copper foil using CVD method. 
hexagonal lattice constant is $\sim 590 \mathrm{~nm}$ in accordance with the NIL mold. The array pattern was well transferred to NIL resist as shown in the inset of Fig. 1(a). After reactive-ion etching (RIE) of graphene holes with $\mathrm{O}_{2}$ plasma and removal of the resist, the diameter of holes increased slightly to $260 \pm 15 \mathrm{~nm}$. Figs. 2(c) and 2(d) depict an atomic force microscopy (AFM) image and a line-scan, respectively, of a GNAs after $\mathrm{Ar} / \mathrm{H}_{2}$ annealing at $400{ }^{\circ} \mathrm{C}$ for $1 \mathrm{~h}$, which can help to remove polymer residue. ${ }^{18}$ The roughness of surface of the remaining graphene indicates the presence of residual material on graphene, which could be due to incomplete removal of PMMA or NIL resist. This may also explain the observation of slightly larger than expected graphene thickness of $\sim 2.6 \pm 0.6 \mathrm{~nm}$ shown in Fig. 2(d) between two markers.

The optical transmittance spectra for 1- and 4-layer graphene and GNAs samples are shown in Fig. 3(a). The 1-layer graphene was found to reduce transmittance at $550 \mathrm{~nm}$ by $4.8 \pm 0.6 \%$, followed by additional reductions of $3.7 \pm 0.5 \%$, $1.8 \pm 0.3 \%$, and $2.7 \pm 0.5 \%$ for 2,3 , and 4 graphene layers, respectively. The variability in light absorption from each layer may be due to imperfections in graphene films used in each layer. These results are consistent with previously reported transmittance on stacked CVD-grown graphene layers ${ }^{11}$ in which an attenuation coefficient of $2.6 \%$ per layer is extracted by fitting the data to Beer's law. Importantly, the transmittance of the layered graphene films does not decrease at longer wavelengths in the same way that ITO does and $R_{S}$ increases with increasing layers of graphene, which could provide improved performance as a transparent conductor in broad-band optoelectronic devices.

The transmittance of the 1- and 4-layer GNAs is, respectively, $97.0 \%$ and $89.5 \%$ at $550 \mathrm{~nm}$, which are $2.0 \%$ and $3.5 \%$ higher than their unpatterned graphene counterparts. At $750 \mathrm{~nm}$, the difference is increased to $3.0 \%$ and $5.0 \%$, respectively. Geometrically, the nanohole array decreases the area of graphene by a factor of 0.16 , which is expected to result in an improvement of less than $1 \%(0.16 \times 5.0 \%$ absorption $=0.8 \%$ absorption reduction) based simply on the decrease in the coverage of GNAs on the substrate compared to unpatterned graphene. The fact that improvement in trans-
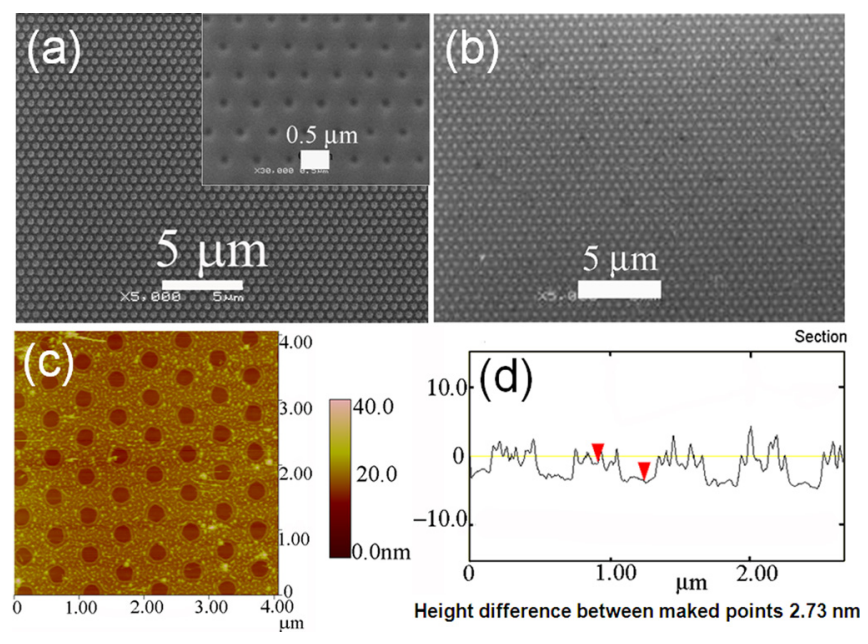

FIG. 2. (Color online) SEM and AFM images of GNAs: (a) GNAs after RIE, before PMMA removal (inset: the same sample before RIE); (b) after PMMA removal with acetone followed with hydrogen/argon annealing; (c) AFM image of and (d) line-scan on GNAs after the annealing.
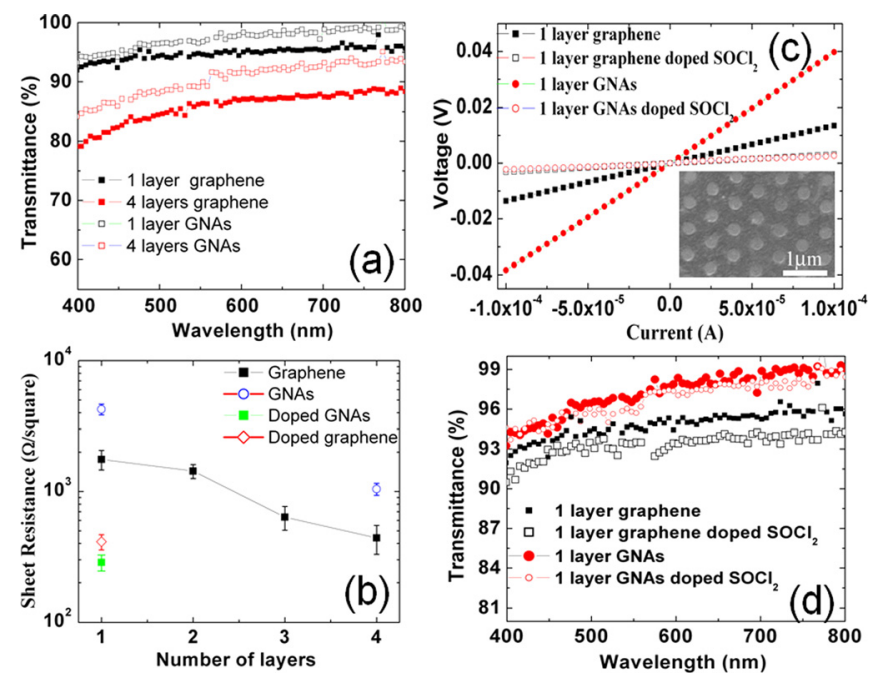

FIG. 3. (Color online) Transmittance spectra of (a) 1- and 4-layer graphene before and after the nanohole array was generated; (b) $\mathrm{R}_{\mathrm{s}}$ of samples shown in Figure 3(a) that of those after doped in $\mathrm{SOCl}_{2}$; (c) $\mathrm{I}$ - $\mathrm{V}$ curves of 1-layer unpatterned graphene and GNAs before and after doping (inset: GNAs sample after doping) and (d) transmittance spectra of the same four samples.

mission is larger than expected based simply on geometrical arguments and wavelength dependence suggests that improved transmittance may not be caused solely by the reduction of graphene coverage. A possible explanation is the interference of nanohole edge with incident light, which may modify the light reflection in a favorable way. Further investigation is underway to pinpoint the mechanism.

The patterning might also affect the electrical properties of graphene. $R_{S}$ of graphene films comprised of various layers is shown in Fig. 3(b). $R_{s}$ of samples was measured in a fourprobe configuration. The width between two electrodes is about $3.2 \mu \mathrm{m}$. The measured range of $1450-2150 \Omega / \square$ for a layer graphene is consistent with that reported by Li et al. ${ }^{11}$ and Verma et al., ${ }^{12}$ but considerably higher than that measured by Bae et al., ${ }^{9}$ even though a similar processing condition was adopted in this experiment. One possible explanation is differences in the size of grains and grain boundaries in graphene. The resistivity would be reduced as grain size is increased. ${ }^{9}$ On the other hand, the interface between graphene and substrate may affect the electron transport via introducing additional charge scattering ${ }^{19}$ or possibly interface doping. ${ }^{20}$ Li et al. reported $\sim 350 \Omega / \square$ for CVD graphene after transferred from $\mathrm{Cu}$ substrate to PMMA and $2100 \Omega / \square$ to glass. ${ }^{11}$

A consistent monotonic drop in $\mathrm{R}_{\mathrm{s}}$, as the number of unpatterned graphene layers increases, can also be observed in Fig. 3(b), as well as a decrease in the $R_{s}$ between the 1layer and 4-layer GNAs. For unpatterned graphene, the $\mathrm{R}_{\mathrm{s}}$ decreases are factors of 1.2, 2.8, and 4.0, respectively, for 2-, 3 -, and 4-layers from that of the single-layer graphene. If the additional layers only increase the cross sectional area available for the charge transport, the $\mathrm{R}_{\mathrm{s}}$ is expected to be reduced by a factor of 2,3 , and 4 from that of the single-layer graphene, respectively. The observed discrepancy may be due to non-uniformity in the CVD graphene resulting from various defects and impurities generated during graphene growth and transfer. Also, the electrodes do not contact each layer equally, and charge transport between layers is not as good as along a given layer. 
The $R_{s}$ of GNAs samples increases considerably compared to unpatterned graphene samples of the same layer thickness for single-layer and 4-layer GNAs, as shown in Fig. 3(b). For the former, $\mathrm{R}_{\mathrm{s}}$ increases by $140 \%$ as compared to that of the unpatterned single-layer graphene, while for the latter it increases by $100 \%$. For the inconsistency of ohmic law (data not shown), it appears the effect of the scattering of electrons by holes. Distinct edge effects have been observed previously in graphene nanoribbons or nanomesh in which the electron pathway widths are $5-15 \mathrm{~nm} .^{21,22}$ The width of between neighboring holes of GNAs is on the order of $300 \mathrm{~nm}$, which is more than an order of magnitude larger than the width of graphene ribbons $(10 \mathrm{~nm})$ with distinctive edge effect on electronic structures. ${ }^{22}$ These data show that the creation of GNAs does in fact increase the resistance of graphene above the level of the original graphene sample. However, the inclusion of hole patterns allows the possibility to tune the resistance of GNAs samples.

In principle, the edges of nanopatterned holes provide sites for chemical modification of graphene that could alter electrical properties, while maintaining the improved optical transmittance of the patterned samples. Fig. 3(c) compares the $I-V$ curves for a single-layer unpatterned graphene and a GNAs before and after $\mathrm{CD}$ in $\mathrm{SOCl}_{2}$. Although increased electrical conductivity is observed for both types of samples, GNAs conductivity increased by 15-18 times upon doping while unpatterned graphene conductivity only increased by 2-4 times. The increase in conductivity of unpatterned graphene is consistent with the previously reported 2-5 times increase of conductivity accompanying p-doping of graphene and carbon nanotubes by attachment of acyl chlorides. ${ }^{13,23} \mathrm{~A}$ similar enhancement of conductivity was reported in $\mathrm{HNO}_{3}$ doped CVD graphene, while it is unclear why the doping effect occurs only to dry-transferred graphene samples. ${ }^{9}$ If dry-transfer causes some defects on graphene, which is supported by the higher $R_{\mathrm{S}}$ on these samples as compared to the wet-transferred counterparts, ${ }^{11}$ the exposed edges simply provide sites for molecule attachment. This argument is supported by the observation of larger enhancement of conductivity by a factor of 5 in graphene flakes. ${ }^{15}$ If a similar doping mechanism applies to GNAs, the large number of edges created on the nanohole arrays could facilitate attachment of $\mathrm{SOCl}_{2}$, which explains the larger enhancement in conductivity observed in doped GNAs samples. In addition, from the SEM images (Fig. 3(c) inset: GNAs sample after doping), we can observe an ordered hexagonal pattern of holes after doping with $\mathrm{SOCl}_{2}$, indicating that the doping treatment does not influence the pattern structure of GNAs. Fig. 3(d) compares the transmittance of four samples. Interestingly, only a slight decrease in transmittance was observed after the doping GNAs while a change was observed in unpatterned graphene. For example, at $550 \mathrm{~nm}$, the transmittance decreased by $1.0 \%$ in GNAs after doping while it decreased by $2.0 \%$ in unpatterned graphene. One possible explanation for the lack of such a correlation between the light transmittance and electrical conductivity in GNAs may be attributed to the spatial non-uniformity of the doping effect. In addition, the light transmission through the nanohole areas is only minimally affected by CD. Therefore, GNAs provide a viable scheme for tuning both light transmittance and electrical conductivity in a favorable way and optimal transparent graphene conductors may be obtained via control of GNAs parameters.

In summary, the fabrication of graphene nanohole array has been demonstrated using nanoimprint lithography. The improved light transmittance of $97 \%$ at $550 \mathrm{~nm}$ and $99 \%$ at $750 \mathrm{~nm}$ for 1-layer GNAs results in part from the reduction of the effective graphene coverage on the patterned sample and the interaction of the light with GNAs. The availability of large number of nanohole edges provides ideal sites for chemical attachment. This leads to reduction of the $R_{s}$ upon doping with $\mathrm{SOCl}_{2}$. These results show that GNAs are a promising approach for tuning the optical and electrical properties of graphene for photovoltaic applications.

J.L. and G.X. contributed equally to this work. The authors acknowledge support from NSF EPSCoR-0903806 for this work. J.W. was also supported in part by ARO Contract No. ARO-W911NF-09-1-0295 and NSF Contract No. NSF-DMR-0803149, and Kansas Technology Enterprise Corporation. C.R. acknowledges a NSF Graduate Research Fellowship. Use of Raman instrumentation at Argonne's Center for Nanoscale Materials was supported by the USDOE, Office of Science, and Office of Basic Energy Sciences. The work performed at Argonne National Laboratory was carried out under Contract No. DE-AC02-06CH11357 between UChicago Argonne, LLC and the USDOE.

${ }^{1}$ K. S. Novoselov et al., Science 306(5296), 666 (2004).

${ }^{2}$ J. B. Wu et al., Appl. Phys. Lett. 92(26), 263302 (2008).

${ }^{3}$ J. K. Wassei and R. B. Kaner, Mater. Today 13(3), 52 (2010).

${ }^{4}$ S. De and J. N. Coleman, ACS Nano 4(5), 2713 (2010).

${ }^{5}$ S. Stankovich et al., Nature 442(7100), 282 (2006).

${ }^{6}$ R. Czerw et al., Phys. Rev. B 66(3), 033480 (2002).

${ }^{7}$ R. R. Nair et al., Science 320(5881), 1308 (2008).

${ }^{8}$ K. S. Novoselov et al., Nature 438(7065), 197 (2005).

${ }^{9}$ S. Bae et al., Nat. Nanotechnol. 5(8), 574 (2010).

${ }^{10}$ X. S. Li et al., Science 324(5932), 1312 (2009).

${ }^{11}$ X. S. Li et al., Nano Lett. 9(12), 4359 (2009).

${ }^{12}$ V. P. Verma et al., Appl. Phys. Lett. 96(20), 203108 (2010).

${ }^{13}$ R. Jackson et al., Adv. Funct. Mater. 18(17), 2548 (2008).

${ }^{14}$ U. Dettlaff-Weglikowska et al., J. Am. Chem. Soc. 127(14), 5125 (2005); W. Zhou et al., Phys. Rev. B 71(20), 205423 (2005).

${ }^{15}$ G. Eda et al., Appl. Phys. Lett. 92(23), 233305 (2008).

${ }^{16}$ A. C. Ferrari et al., Phys. Rev. Lett. 97(18), 187401 (2006).

${ }^{17}$ I. Calizo et al., J. Appl. Phys. 106(4), 043509 (2009).

${ }^{18}$ M. Ishigami et al., Nano Lett. 7(6), 1643 (2007).

${ }^{19}$ J. H. Chen et al., Nat. Nanotechnol. 3(4), 206 (2008).

${ }^{20}$ K. K. Kim et al., Nanotechnology 21(28), 285205 (2010).

${ }^{21}$ X. G. Liang et al., Nano Lett. 10(7), 2454 (2010).

${ }^{22}$ X. R. Wang et al., Phys. Rev. Lett. 100(20), 206803 (2008).

${ }^{23}$ V. C. Tung et al., Nano Lett. 9(5), 1949 (2009).

${ }^{24}$ See supplementary material at http://dx.doi.org/10.1063/1.3610939 for the summary of transmittance at $550 \mathrm{~nm}$ and $750 \mathrm{~nm}$, respectively, and the $\mathrm{R}_{\mathrm{s}}$ for graphene and GNAs samples. 